\title{
Lost productivity associated with headache and depression: a quality improvement project identifying a patient population at risk
}

Virginia B. Baker ${ }^{1 *}$, Christopher B. Sowers ${ }^{2}$ and Nawaz K. Hack ${ }^{1}$

\begin{abstract}
Objective: This quality improvement project was implemented in order to highlight the association between headache, mTBI and depression on lost productivity and resource utilization.

Background: Mood disorders, environment and traumatic brain injury are common in patients with headache, and have been shown to influence clinical course, treatment response and outcome. Although widely recognized, the association of these factors on clinical outcomes, resource utilization and productivity is not well understood.

Methods: All patients presenting to a military referral center for migraines are assessed for presence of traumatic brain injury, Headache Impact Score (HIT-6) and Patient Depression Questionnaire (PHQ-9). Based on screening, patients are offered referral to mental health and a multidisciplinary headache education course.

Results: 237 patients were seen for headache or migraine. 180 patients had severely disabling headaches. These patients accounted for 146 emergency room visits over the course of one year. Of headache patients, $65 \%$ met criteria for depression and 15\% of patients had severe depression. Only 37\% of these patients carried a formal diagnosis of depression and 38\% had been seen by mental health. Lost productivity and duty limitations were significantly associated with severity of depression. In service members screening positively for mild, moderate or severe depression, duty restrictions had been placed on $8.3 \%, 32.5 \%$ and $53.8 \%$, respectively. Only $3.8 \%$ of patients who did not screen for depression had similar duty limitations. A history of mTBI strongly correlated with comorbid depression. Lost productivity and duty limitations were not impacted by other headache characteristics or HIT-6 scores.

Conclusions: This quality improvement project identified a practice gap for treatment of comorbid depression in patients presenting to Neurology for headache. Depression strongly correlated with productivity loss, highlighting a possible target for the economic burden of headache.
\end{abstract}

Keywords: Migraine, PHQ-9, Multi-disciplinary, Productivity, Mild traumatic brain injury (mTBI)

\footnotetext{
* Correspondence: Ginni.baker11@gmail.com

${ }^{1}$ Walter Reed National Military Medical Center, Neurology Department, 8901

Rockville Pike, Bethesda, MD 20889, USA

Full list of author information is available at the end of the article
}

\section{$\triangle B M C$}

C C The Author(s). 2020 Open Access This article is licensed under a Creative Commons Attribution 4.0 International License, which permits use, sharing, adaptation, distribution and reproduction in any medium or format, as long as you give appropriate credit to the original author(s) and the source, provide a link to the Creative Commons licence, and indicate if changes were made. The images or other third party material in this article are included in the article's Creative Commons licence, unless indicated otherwise in a credit line to the material. If material is not included in the article's Creative Commons licence and your intended use is not permitted by statutory regulation or exceeds the permitted use, you will need to obtain permission directly from the copyright holder. To view a copy of this licence, visit http://creativecommons.org/licenses/by/4.0/ The Creative Commons Public Domain Dedication waiver (http://creativecommons.org/publicdomain/zero/1.0/) applies to the data made available in this article, unless otherwise stated in a credit line to the data. 


\section{Background}

Migraines and headaches affect one in seven Americans annually [1, 2]. In 2010, headache accounted for greater than $3 \%$ of all emergency room visits, and was documented as the fourth leading cause of visits to the emergency department within the United States [1]. This has considerable financial implications, through utilization of healthcare resources and lost productivity in the workplace [3, 4]. Indirect costs, including disability, lost productivity and absence from work account for more than $50-70 \%$ of the economic burden from headaches [5-7]. Within the military, headache accounts for greater than $50 \%$ of outpatient Neurology encounters. It is a cause for medical attention in $4 \%$ of all service members, placing significant demands on the healthcare system [8].

Mood disorders are common in the headache population, and have been shown to influence clinical course, treatment response and outcome [9-12]. Patients with migraine are 2-4 times more likely to develop major depressive disorder $[13,14]$. It is theorized that environmental stressors, work demands, changing sleep cycles and the frequency of traumatic brain injury magnify the burden of headaches and psychiatric conditions [15-18]. Furthermore, major depressive disorders and pain syndromes have biological associations with levels of thyroid hormones, prolactin and serotonin. These markers may also be associated with risk for suicide [19]. Suicide has far-reaching psychological implications for not only the patient, but also his or her successors, leading to significant morbidity, mortality and use of healthcare resources [20]. Despite this recognized association and grave impact, the relationship between headaches and depression is not well understood [21-25].

Concurrent treatment of both headache and depression is supported through growing research and literature [26, 27]. Our Quality Improvement project initiated at Walter Reed National Military Medical Center (WRNMMC) instituted a screening tool to better understand the relationship between headache, psychiatric comorbidities and productivity. This was further stratified by the presence of mild traumatic brain injury (mTBI). Neurology promoted a multidisciplinary approach to care through group education and interdisciplinary involvement $[24,28]$. This strategy was implemented to decrease resource utilization and loss of productivity among service members and their dependents. This is the first paper to investigate the interplay between migraines, mTBI and depression on lost productivity in the United States [29, 30].

\section{Methods}

From August 2018 until June 2019, an IRB-approved Quality Improvement Project was initiated at Walter
Reed National Military Medical Center (WRNMMC) in order to screen for and treat co-morbidities in patients presenting for headache. All patients presenting to the Neurology clinic for a diagnosis of headache or migraine were administered a headache intake questionnaire. Exclusion criteria included patients with a diagnosis of a secondary headache disorder or a history of moderate or severe traumatic brain injury. Only patients who are active duty, active duty dependents or military retirees are eligible for care at WRNMMC.

Headache intake questionnaires included the Headache Impact Test (HIT-6) and Patient Health Questionnaire (PHQ-9). HIT-6 score is a validated tool for headache, including migraine and chronic migraine. Scores range from 36 to 78 and correlate with the level of impact on daily life and ability to function. Scores greater than or equal to 60 indicate a severe impact on patient life [31, 32]. The PHQ-9 is a validated, selfadministered scoring system used to screen for depression. Scores range from 0 to 29 , with a score greater or equal than $5,10,15$, and 20 representing mild, moderate, moderately severe and severe depression, respectively $[33,34]$. Scores greater than or equal to 10 have been shown to have a sensitivity of .88 and specificity of .85 for a diagnosis of Major Depressive Disorder [35]. In addition, we surveyed utilization of healthcare resources through hospital admissions and emergency room visits. Productivity was assessed through documentation of duty restrictions, limited duty status or medical separation from the military due to headache or migraine. Financial implications were estimated based on a computational analysis by the National Defense Research Institute (RAND) published in 2007. This analysis calculated individual costs based on active duty pay, benefits and retiree compensation [36].

Patients were evaluated for medical and psychiatric comorbidities as well as utilization of healthcare resources using intake forms and physician interview. Based on this information, referral to mental health was offered and a migraine education class was encouraged. The migraine education class emphasized a multidisciplinary approach to care, utilizing mental health, psychiatry, physical therapy and sleep therapy counterparts. It stressed the importance of lifestyle modifications in migraine prevention and management, to include stress reduction, trigger avoidance and implementation of healthy routines including scheduled hydration, exercise and sleep. Referrals to psychiatry were also encouraged for all patients who screened positively for depression. However, the specifics of treatment and management of these psychiatric comorbidities were not reviewed. This project was implemented to increase recognition and improve access to care for comorbid psychiatric conditions in headache patients. Ultimately, we hoped to 
highlight that the prevalence of depression and insufficient access to mental health services had a negative impact on resource utilization.

\section{Results}

From August 2018 until June 2019, 237 patients were seen in a Neurology tertiary care center for headache or migraine and 208 completed depression screening. Of these patients, $77 \%$ were active duty or retired military personnel. No patients evaluated had a history of moderate or severe traumatic brain injury or secondary headache disorder. $32 \%$ had a history of a prior mild traumatic brain injury (mTBI). The population averaged 16 headache days per month. Average headache duration was $15 \mathrm{~h}$ (Table 1). Headache impact score averaged 64 points, indicating a severe impact on patient life. 180 patients had severely disabling headaches. Of the 174 active duty personnel, 36 were undergoing a medical board for headaches, meaning that they were no longer qualified to perform as required for military duty. Over the course of one year, this population accounted for 146 emergency room visits for acute treatment of headache or migraine.

In the headache population, average PHQ-9 selfassessment score was 8 , consistent with a diagnosis of mild depression. PHQ-9 scores were positive for depression in $65 \%$ of screened patients presenting for a primary headache disorder. Of patients who met criteria, 37\%

Table 1 Patient demographics. A comparison of duty status, headache diagnosis, TBI history and medication use in patients who screened positively versus negatively for depression

\begin{tabular}{lll}
\hline Characteristic & No Depression (73) & Depression (135) \\
\hline Duty Status - no. (\%) & & \\
Active & $38(52 \%)$ & $67(50 \%)$ \\
Retired & $21(29 \%)$ & $34(25 \%)$ \\
Dependent & $14(19 \%)$ & $34(25 \%)$ \\
Diagnosis - no. (\%) & & \\
PTHA & $8(11 \%)$ & $40(30 \%)$ \\
Total Migraine & $65(89 \%)$ & $95(70 \%)$ \\
$\quad$ Episodic Migraine & $39(53.4 \%)$ & $33(24 \%)$ \\
$\quad$ Chronic Migraine & $2(35.6 \%)$ & $55(46 \%)$ \\
Mild TBI - no. (\%) & & 18 \\
History mTBl & $11(15 \%)$ & 17.7 \\
Headache Characteristics & & $73(54 \%)$ \\
Frequency - day / mo. & 12 & $80(59 \%)$ \\
Duration - hrs & 10.2 & $15(89 \%)$ \\
Medication Use - no. (\%) & & \\
BOTOX & $32(44 \%)$ & $36(49 \%)$ \\
Taking Oral Preventatives & $69(95 \%)$ & \\
Taking Oral Abortives & & \\
\hline
\end{tabular}

had a formal diagnosis of depression made by a medical provider and $38 \%$ had been engaged with mental health at the time of presentation to Neurology (Fig. 1). 15\% of patients met criteria for severe depression. Of patients meeting criteria for severe depression, $71 \%$ had a documented diagnosis and $68 \%$ of these patients were engaged with mental health. Depression was more likely in patients who had a history of prior mTBI, with a prevalence of $81 \%$. Of patients with a history of $\mathrm{mTBI}$ and depression, $49 \%$ had a diagnosis and $45 \%$ were engaged with mental health services. Post-traumatic stress disorder was documented in $14 \%$ of this patient population.

Patients were stratified based on the presence and severity of depression, and statistical analysis was performed using chi-squared and ANOVA tests. There was no significant difference in headache characteristics or likelihood to present to the emergency room for headache treatment. Headache impact test (HIT-6) averaged 61 in patients without depression. Patients with mild, moderate and severe depression had a HIT-6 score of 63, 67 and 67 respectively. There was positive trend in HIT-6 score based on depression severity, but this did not meet statistical significance (supplemental figure 1). History of mTBI did not impact headache days, headache duration or HIT-6 score, but did correlate with higher depression rates.

Patients with higher depression scores were significantly more likely to have active duty work restrictions or be medically boarded for migraines. Duty restrictions placed as a result of headache or migraine occurred in $3.8 \%$ of patients who did not meet criteria for depression. In patients with depression, $29 \%$ had similar duty restrictions. In patients scoring for mild, moderate or severe depression, duty restrictions had been placed on $8.3 \%, 32.5 \%$ and $53.8 \%$, respectively (Fig. 2). Patients with higher severity of depression had a significant increase in the number of medical boards and duty limitations as a result of headache. Of all patients undergoing a medical board or limited duty restrictions for headaches, 94\% had a PHQ-9 score consistent with a diagnosis of depression. There was no change in the number of medical boards or duty limitations based on HIT-6 scoring system or other headache characteristics including headache days or duration.

\section{Discussion}

Twenty percent of our active duty cohort was facing duty restrictions with potential for medical separation. Based on RAND and Secretary of Defense estimates, an enlisted service member's military separation accounts for a lifecycle cost of greater than $\$ 860,000$ and an officer member's costs account for greater than $\$ 1,700,000$ [36]. Medical boards within our cohort accounted for at least 31 million dollars of resource utilization and lost 


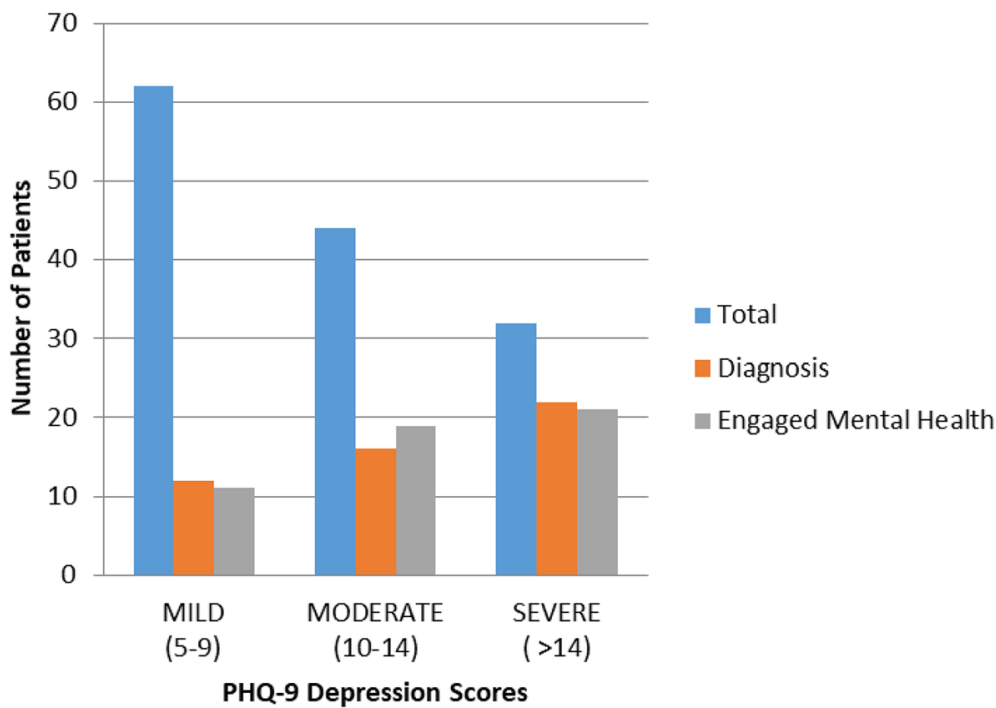

Fig. 1 Practice gap in patients screening positively for depression. Patients who screened positively on PHQ-9 for depression were evaluated for a formal diagnosis made by a medical provider and for engagement of mental health services

productivity. Of service members who screened positive for depression, nearly $30 \%$ were facing duty restrictions or medical separation from the military. Interestingly, of members who did not screen positive for depression, only $3.8 \%$ had similar duty restrictions. The military provides a unique patient population for early detection of lost productivity, given required reporting of medicallyrelated duty limitations. This provides a quantifiable metric for lost productivity and career limitations and identifies possible improvements for clinical practice.
Our study identifies comorbid depression as a possible target for the lost productivity, career impact and substantial financial implications as a result of headache.

Although it is recognized that headaches and migraines are a major cause for lost productivity and increased utilization of resources, this has not been evaluated concurrently with depression [37, 38]. In this patient population, $65 \%$ screened positive for depression and $15 \%$ met criteria for severe depression. Military duty restrictions and lost productivity correlate greater with

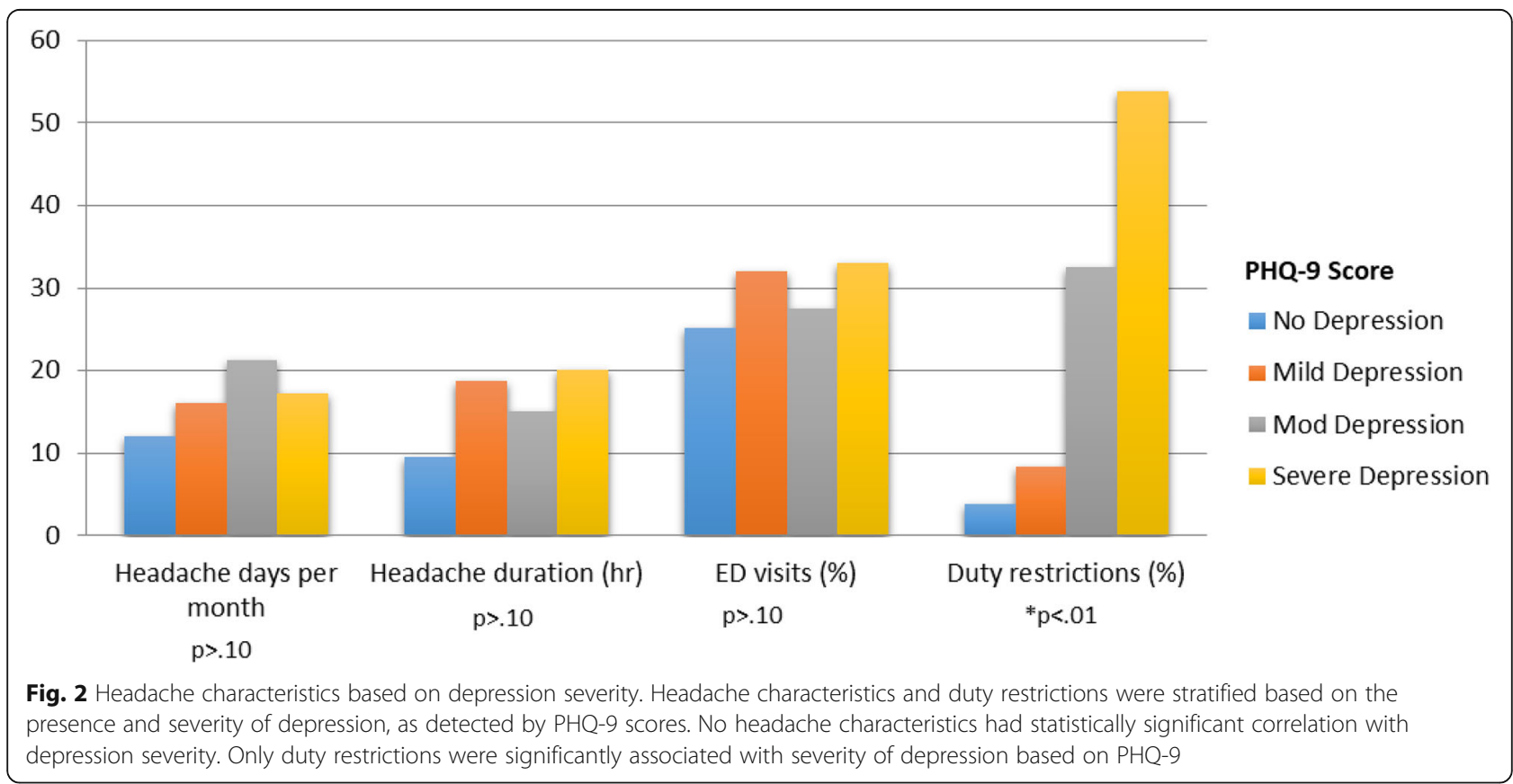


depression than with headache impact scores, despite patients presenting for a primary complaint of headache. A limitation of this manuscript includes the lack of information regarding comorbid anxiety, which is also known to have high correlation with migraine and headaches. In future research, we recommend also utilizing a screening tool for anxiety, such as the Generalized Anxiety Disorder (GAD-7). Presence of post-traumatic stress disorder in $14 \%$ of our patient population may indicate that comorbid anxiety and arousal conditions were coexisting.

Less than half of patients who scored positive on PHQ-9 had a mental health diagnosis and even fewer were engaged with mental health services. Recognition and treatment were more likely if patients met criteria for severe depression. Even in this subgroup, nearly onethird were without a formal diagnosis or engaged with mental health. This practice gap presents an opportunity for Neurologists to improve patient safety, quality of life and productivity. We propose that all patients presenting to Neurology for treatment of headache or migraine be screened for psychiatric comorbidities. Patients who screen positively should be referred to behavioral health or psychiatry for early evaluation and treatment. Our screening system promotes a multimodal approach to care which improved access to mental health services in patients presenting for headaches. We believe that early recognition and treatment of depression may improve productivity and maintain deployability of our armed forces and their dependents [39].

A limitation of this data is that the results may not be generalizable to the United States population as a whole. Our population had been exposed to unique environmental and physical factors, including changing environments, inconsistent schedules, atypical work conditions and higher frequency of traumatic brain injuries. These factors have been shown to affect headaches and migraines, and may predispose patients to higher PHQ-9 or HIT-6 scores. In our subgroup analysis, traumatic brain injury was independently associated with both depression and lost productivity. One-third of patients presenting for headache or migraine had a diagnosis of prior mild traumatic brain injury. Patients with a history of traumatic brain injury were more likely to meet criteria for depression, which may contribute to the loss of productivity or limitations of duty in this population. The association of traumatic brain injury with heightened PHQ-9 scores highlights the impact of militaryspecific stressors on patient outcomes.

This Quality Improvement project can serve as a proof of concept for future research and a possible target for growing healthcare costs. This small cohort accounted for a revenue loss of 31 million dollars. The estimated cost to the United States healthcare system would be in the billions [6]. Treatment of comorbid psychiatric conditions may have considerable financial implications in the headache population, especially within the United States military population. The study utilized a standardized and validated screening tool to detect comorbid psychiatric conditions in patients presenting for headache. Through recognition of comorbid depression, access to care for mental health services was improved. Further research is needed to define the effect of treating depression on productivity and healthcare costs in patients with headache or migraine.

\section{Conclusions}

This study is the first to present the relationship between migraine, depression and traumatic brain injury on lost productivity. We propose a concept model for all Neurology clinics, which utilizes screening tools as a basis for mental health referrals and involvement in a multidisciplinary headache education course. In the headache population, depression appears to have a high predictive value for productivity loss. Early treatment and detection can improve quality of life, enhance productivity and decrease the use of medical resources.

\section{Supplementary information}

Supplementary information accompanies this paper at https://doi.org/10 1186/s10194-020-01107-4.

Additional file 1: Supplemental figure 1. Correlation of HIT-6 score with severity of depression. Although not statistically significant, Headache Impact Test (HIT-6) had a correlation with the severity of depression.

\section{Abbreviations}

HIT-6: Headache Impact Test; PHQ-9: Public Health Questionnaire; TBI: Traumatic brain injury; mTBI: mild traumatic brain injury; WRNMMC: Walter Reed National Military Medical Center

\section{Acknowledgements}

Not applicable.

\section{Authors' contributions}

Virginia B. Baker contributions include data acquisition, analysis and interpretation. She performed statistics for data analysis and is the primary author of manuscript and abstracts. Christopher B. Sowers contributions include data review, creation of figures and manuscript edits. Nawaz K. Hack contributions include project design and data acquisition, secondary manuscript author and primary manuscript editor. The authors read and approved the final manuscript. The principal author takes full responsibility for the data presented in this study, analysis of the data, conclusions, and conduct of the research. The principal author had full access to those data and has maintained the right to publish any and all data independent of any third party.

\section{Funding}

The authors received no financial support for the research, authorship, and/ or publication of this article.

Availability of data and materials

Available under request from the corresponding author. 


\section{Ethics approval and consent to participate}

IRB approval with determination of not research by Walter Reed National Military Medical Center. WRNMMC-EDO-2018-0210.

\section{Consent for publication}

Provided by all authors included on this manuscript.

The views expressed in this chapter are those of the author and do not reflect the official policy of the Department of Army/Navy/Air Force, Department of Defense, or U.S. Government. This research does not reflect the views or opinions of the United States Military or Government.

\section{Competing interests}

No competing interests for this manuscript. The views expressed in this chapter are those of the author and do not reflect the official policy of the Department of Army/Navy/Air Force, Department of Defense, or U.S. Government. This does not reflect the views or opinions of the United States Military or Government.

\section{Author details}

${ }^{1}$ Walter Reed National Military Medical Center, Neurology Department, 8901 Rockville Pike, Bethesda, MD 20889, USA. ${ }^{2}$ Naval Medical Center San Diego, Anesthesiology Department, San Diego, CA, USA.

Received: 8 February 2020 Accepted: 13 April 2020

Published online: 11 May 2020

\section{References}

1. Burch RC et al (2015) The prevalence and burden of migraine and severe headache in the United States: updated statistics from government health surveillance studies. Headache 55(1):21-34

2. Smitherman TA et al (2013) The prevalence, impact, and treatment of migraine and severe headaches in the United States: a review of statistics from national surveillance studies. Headache 53(3):427-436

3. Hazard E et al (2009) The burden of migraine in the United States: current and emerging perspectives on disease management and economic analysis. Value Health 12(1):55-64

4. Elston Lafata J et al (2004) The medical care utilization and costs associated with migraine headache. J Gen Intern Med 19(10):1005-1012

5. Porter JK et al (2019) Costs of acute headache medication use and productivity losses among patients with migraine: insights from three randomized controlled trials. Pharmacoecon Open 3(3):411-417

6. Bonafede $\mathrm{M}$ et al (2018) Direct and indirect healthcare resource utilization and costs among migraine patients in the United States. Headache 58(5): 700-714

7. Stokes M et al (2011) Cost of health care among patients with chronic and episodic migraine in Canada and the USA: results from the international burden of migraine study (IBMS). Headache 51(7):1058-1077

8. Armed Forces Health Surveillance Center (AFHSC (2012). Outpatient encounters associated with diagnostic codes for migraine and other types of headaches, active component service members, 1998-2010. MSMR, 19(2), 12.

9. May A, Schulte LH (2016) Chronic migraine: risk factors, mechanisms and treatment. Nat Rev Neurol 12(8):455-464

10. Peres MFP et al (2017) Anxiety and depression symptoms and migraine: a symptom-based approach research. J Headache Pain 18(1):37-37

11. Guillem E, Pelissolo A, Lepine JP (1999) Mental disorders and migraine: epidemiologic studies. Encephale 25(5):436-442

12. Shala N, Dreshaj S (2018) Association of depression, anxiety and posttraumatic stress disorder with migraine: data from Kosovo. Neurol Neurochir Pol 52(4):490-494

13. Amoozegar F (2017) Depression comorbidity in migraine. Int Rev Psychiatry 29(5):504-515

14. Minen MT et al (2016) Migraine and its psychiatric comorbidities. J Neurol Neurosurg Psychiatry 87(7):741-749

15. Skopp NA et al (2012) Relations between suicide and traumatic brain injury, psychiatric diagnoses, and relationship problems, active component, U.S. armed forces, 2001-2009. Msmr 19(2):7-11

16. Couch JR, Stewart KE (2016) Headache prevalence at 4-11 years after deployment-related traumatic brain injury in veterans of Iraq and Afghanistan wars and comparison to controls: a matched case-controlled study. Headache 56(6):1004-1021
17. Fraser F et al (2017) Behavioral treatments for post-traumatic headache. Curr Pain Headache Rep 21(5):22

18. Nampiaparampil DE (2008) Prevalence of chronic pain after traumatic brain injury: a systematic review. Jama 300(6):711-719

19. Pompili $\mathrm{M}$ et al (2012) Prolactin and thyroid hormone levels are associated with suicide attempts in psychiatric patients. Psychiatry Res 200(2-3):389394

20. Pompili $\mathrm{M}$ et al (2013) Bereavement after the suicide of a significant other. Indian J Psychiatry 55(3):256-263

21. Bair MJ et al (2003) Depression and pain comorbidity: a literature review. Arch Intern Med 163(20):2433-2445

22. Ziegler DK (1993) Headache and depression: serotonin pathways as a common clue. Arch Neurol 50(5):449-449

23. Louter MA et al (2015) Symptom dimensions of affective disorders in migraine patients. J Psychosom Res 79(5):458-463

24. Silberstein SD (1998) Comprehensive management of headache and depression. Cephalalgia 18(Suppl 21):50-55

25. Yang $Y$ et al (2016) Genetic epidemiology of migraine and depression. Cephalalgia 36(7):679-691

26. Wallasch TM, Angeli A, Kropp P (2012) Outcomes of a headache-specific cross-sectional multidisciplinary treatment program. Headache 52(7):10941105

27. Silberstein SD, Lipton RB, Breslau N (1995) Migraine: association with personality characteristics and psychopathology. Cephalalgia 15(5):358-369 discussion 336

28. Diener HC et al (2011) Integrated headache care. Cephalalgia 31(9):10391047

29. Lucas $S$ et al (2016) Comorbidity of headache and depression after mild traumatic brain injury. Headache 56(2):323-330

30. Jaramillo CA et al (2016) A cohort study examining headaches among veterans of Iraq and Afghanistan wars: associations with traumatic brain injury, PTSD, and depression. Headache 56(3):528-539

31. Yang $M$ et al (2011) Validation of the headache impact test (HIT-6) across episodic and chronic migraine. Cephalalgia 31(3):357-367

32. Rendas-Baum $R$ et al (2014) Validation of the headache impact test (HIT-6) in patients with chronic migraine. Health Qual Life Outcomes 12:117

33. Kroenke K, Spitzer RL, Williams JB (2001) The PHO-9: validity of a brief depression severity measure. J Gen Intern Med 16(9):606-613

34. Maurer DM, Raymond TJ, Davis BN (2018) Depression: screening and diagnosis. Am Fam Physician 98(8):508-515

35. Levis B, Benedetti A, Thombs BD (2019) Accuracy of patient health Questionnaire-9 (PHQ-9) for screening to detect major depression: individual participant data meta-analysis. Bmj 365:11476

36. Dahlman CJ (2007) The cost of a military person-year: a method for computing savings from force reductions

37. Burton WN et al (2002) The economic burden of lost productivity due to migraine headache: a specific worksite analysis. J Occup Environ Med 44(6): 523-529

38. Lanteri-Minet M (2014) Economic burden and costs of chronic migraine. Curr Pain Headache Rep 18(1):385

39. Minen MT, Loder E, Friedman B (2014) Factors associated with emergency department visits for migraine: an observational study. Headache 54(10): $1611-1618$

\section{Publisher's Note}

Springer Nature remains neutral with regard to jurisdictional claims in published maps and institutional affiliations.

Ready to submit your research? Choose BMC and benefit from:

- fast, convenient online submission

- thorough peer review by experienced researchers in your field

- rapid publication on acceptance

- support for research data, including large and complex data types

- gold Open Access which fosters wider collaboration and increased citations

- maximum visibility for your research: over $100 \mathrm{M}$ website views per year

At BMC, research is always in progress.

Learn more biomedcentral.com/submissions 